\title{
Resource Selection in Grid Environment Based on Trust Evaluation Using Feedback and Performance
}

\author{
${ }^{1}$ Prakash Mohan and ${ }^{2}$ Ravichandran Thangavel \\ ${ }^{1}$ Department of Information Technology, Jeppiaar Institute of Technology, Chennai, India \\ ${ }^{2}$ Department of Computer Science and Engineering, Hindustan Institute of Technology, Coimbatore, India
}

Received 2013-02-05, Revised 2013-05-15; Accepted 2013-07-23

\begin{abstract}
A grid computing is a collection of distributed computing resources owned by many organizations across boundaries and not dedicated for a single user available in the Virtual Organization. Grid computing environment provides more computational capabilities to increase the scalability and efficiency of the infrastructure. Resource selection in computational grid is the open issue for executing complex jobs. Trust is the one of the important factor for selecting the resources in computational grid. We introduced a new technique for finding the trusted resource. In our proposed method, we computed the trusted resource from user's feedback and performance. While selecting the resource, overall trustworthiness is compared. The result of the resource selection algorithm is that most trust resource weight is above the threshold level. In our proposed algorithm job is submitted to the trust resource available in grid.
\end{abstract}

Keywords: Agent, Users Feedback, Resource Selection, Resource Performance, Trust, Trustworthiness, Trust Management, Job Scheduling

\section{INTRODUCTION}

A computational grid is a large scale heterogeneous collection of autonomous systems, geographically (Foster et al., 2001). Recent scientific problems are very complex to solve and they need enormous computation power and storage space. Grid computing, is adapted from Ian Foster, it is concerned with "coordinated resource sharing and problem solving in dynamic, multi-institutional virtual organizations". The huge volume ( $\mathrm{Li}$ and Baker, 2005) of data takes longer time for computing, processing and storing. For computing the huge volume of data the broker has to analyze whether the resource is a trusted resource or not. An important issue while selecting the trusted resource is that the load in grid environment is to be maintained uniformly, on the other hand the entire grid sites are to be balanced. The main purpose of load balancing is to distributed and interconnected with the networks

increase the system performance and resource utilization. Most of the grid sites do not utilize the resource properly and efficiently. The job is submitted to a particular gird site, if the load is not evenly distributed and balanced, this may lead to critical problem. This critical problem in a heterogeneous environment becomes more harmful. To avoid this issue, for executing a job in heterogeneous environment the users have to find the feasible resources or optimal resources. The job has to be submitted, if and only if the resource is trustworthy otherwise the next available optimal resource to be searched for executing the job.

Many researches' issues are in the area of trusted grid scheduling. Wang et al. (2011) proposed a dynamic trust model. This trust model depends on direct and recommendation trust. In this model, the trust is calulcated based on the performance of entity and its history. We have considered the dynamic grid environment with users feedback and resource Corresponding Author: Prakash Mohan, Department of Information Technology, Jeppiaar Institute of Technology, Chennai, India 
performance for calculating the trust. These paramerter are addressed in our work and reviewed.

Can and Bhargava (2013) proposed a Self ORganizing Trust Model (SORT). In this model peers creates its own trust network using local information available. For measuring the trustworthinees of peers in a network is evaluated using two contexts, services and recommendation of contexts.In this point, if the peer changes form one network to other it may lost its trusted network. We have considered the the feedback against the budget and affordability of the user for trustworthiness of a peer.

Bawa and Sharma (2012) proposed a safety mechanism for selecting a resource in grid environment. In this method they have calculated resource reputation based on the users feedback and the Reliability Factor (RF) of the resource site is calculated through self-protection and reputation weightage. We have taken a trust decision model to decide whether the decided resource is trustworthy or not.

In dynamic environment the selection of resource is based on the accumulated trust history. itthis model reputation is calculated using its history. Shun-Fu et al. (2012) proposed a reliablity model for resouce selection based on the Web service frame to satisfy both user and provider. A resource provisioning policy (Aron and Chana, 2012) is used for resource scheduling and resource allocation. The protocol for resouce provisioning is implemented using XML and validated using Z-Formal specification for mininizing the cost and submission burst time. Selecting a resource and submitting a task resource don't suffice, the task to be submitted only to the trusted resource in grid. The trust is found based on the performance and users performance by calculating the resources utilized by the previous jobs more effectively and efficiently.

A new trust managemet system (Siadat et al., 2009) with two levels is proposed to imporve the security in grid. One of the level manages the task available in resource node called Domain Trust Manager (DTM). Ring algorithm which selects one of the DTM resource nodes in a domain. Global Trust Manager (GRM) is located in the upper level used for registration, trust negotiation and initialization. The lower level includes feedback, demand trust valuation, trust evaluation and monitoring. In our proposed method the evolution is made by using the feedback and the performance of the resource. So the resource discovered in our system is trustworthy.

\section{MATERIALS AND METHODS}

\subsection{Trust Resource Selection}

\subsubsection{The Notion of Trust}

The trust is easy to recognize and experience in day to day life, but it is quite taxing to define because it manifests itself in different ways. Generally the literature on the trust is confusing because the term 'trust' signifies a variety of meanings (McKnight and Chervany, 2001). Two commonly used trusts are reliability trust and decision trust.

A reliability trust denotes the performance of other person not on his own. "Reliability trust is the subjective probability by which an individual A expects another individual $\mathrm{B}$, to perform a given action on which its interest depends".

A decision trust is that which is obtained on ones willingness or situation related to security. "Decision trust is the extent to which one party is will to depend on something or somebody in a given situation with a feeling of relative security, even though negative consequences are possible".

\subsection{Our Trust Model}

A major and difficult problem in sharing resources is due to heterogeneity. Whenever two parties want to interact with each other, they should be able to evaluate the amount of trust on each other using some evaluation factors. The evaluation metrics can be considered as the recommendation or feedback given by the user, budget constrain, affordability, availability of the resources, success rate, obviously the trust can be managed in policy based and reputation based approach. The reputation based approach trust contains:

- Users Feedback

- Resource Performance

- Trust decision approach

A trust resource selection model is shown in Fig. 1, in which the user submits a job through grid portal. The agent/broker, which has all the information about the resource, matches the resource according to the user's needs. Once the user's required need is satisfied with the availability of the resource, then all those resources are matched in the trusted database. The final decision of the trustworthiness is calculated by the trust decision whether to submit the job to the particular resource or to submit to the any other resource available in the grid. 


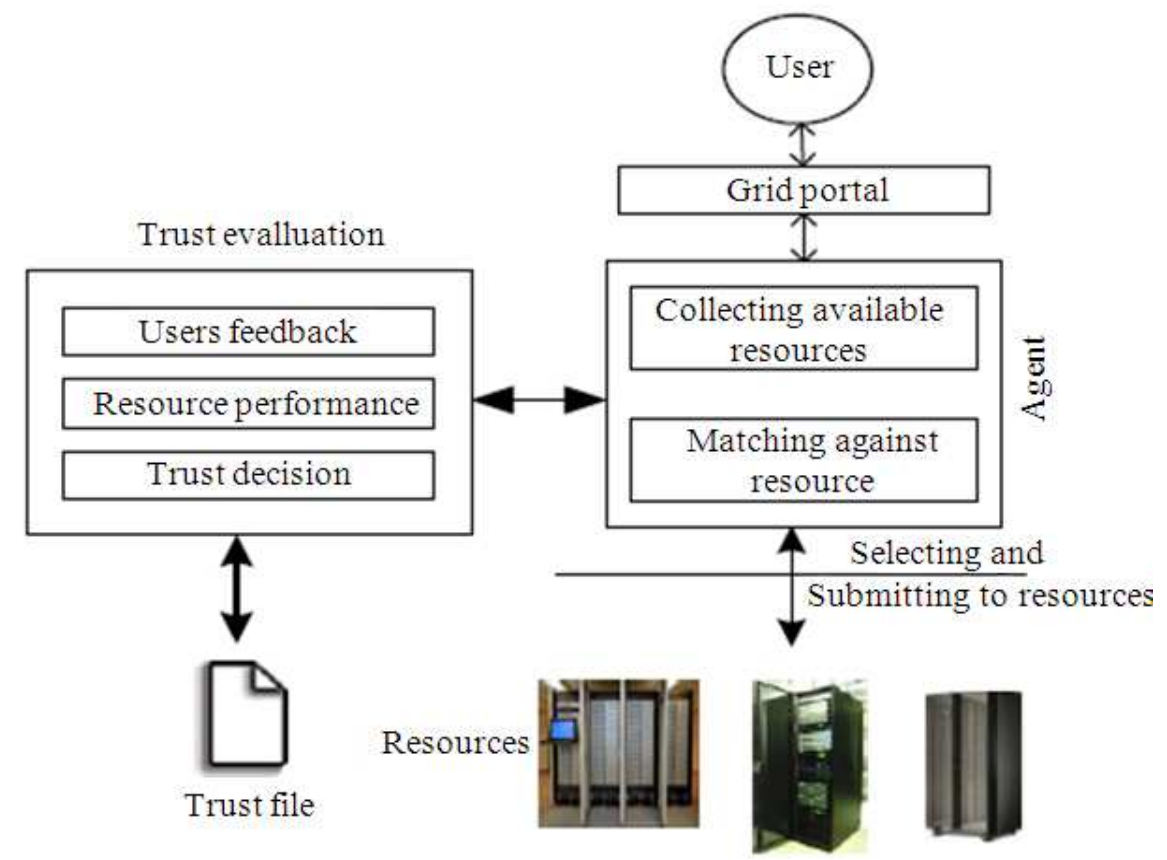

Fig. 1. Trust resource selection model

\subsection{Users Feedback}

The most important approach to find the trusted resource is user's feedback. Once the submitted job is processed and completed the user sends the feedback and it's stored in the trusted database. The collected information are in the scale $[0,1]$. The far most feedback collected from the user is the affordability. The affordability is entirely based on the budget.

The affordability $\mathrm{Aff}_{\mathrm{f}}$ in Equation 1 is calculated by the ration of the budget assigned by the user while submitting the job to the resource provider to the budget committed/assigned by the resource provider, at the time of registration:

$$
\text { Affordability } \operatorname{Aff}_{\mathrm{f}}=\frac{\mathrm{B}_{\mathrm{u}}}{\mathrm{B}_{\mathrm{r}}}
$$

Where:

$\mathrm{B}_{\mathrm{u}}=$ Budget assigned by the user while submitting a job $\mathrm{B}_{\mathrm{r}}=$ Budget committed by the resource provider:

$$
\operatorname{Aff}_{\mathrm{f}}= \begin{cases}<0.25 & \text { trust }=0 \\ >0.25 & \text { trust }=\mathrm{B}_{\mathrm{u}} / \mathrm{B}_{\mathrm{r}}\end{cases}
$$

The feedback taken for this affordability is the ratio of the user budget to the budget committed by the re-source provider or total budget obtained to complete the job.
The availability of the resource is taken as the next feedback. In Equation 2 the availability of the resource is calculated using the total time the resource is idle to the total time the resources are busy for executing the task:

Availability Av $=\frac{R_{\text {idle }}}{R_{\text {busy }}}$

Where:

$\mathrm{R}_{\mathrm{idle}}=$ Total idle time of resource

$\mathrm{R}_{\text {busy }}=$ Total busy time of resource:

$$
\text { Avf }= \begin{cases}>0.75 & \text { neglate the resource } \\ 0.25 \text { to } 0.75 & \text { accept for trust } \\ <0.25 & \text { resource is over loaded }\end{cases}
$$

The success rate is the another important factor for calculating the feedback and its calculated in Equation 3. The ratio of jobs completed by the resource provider and the total number of jobs submitted by the user to resource provider:

Sucess rate $S_{r}=\frac{J_{c}}{J_{s}}$

Where:

$\mathrm{J}_{\mathrm{c}}=$ No. of jobs completed by the resource provider

$\mathrm{J}_{\mathrm{s}}=$ Total no. of job submitted by the resource provider: 


$$
\mathrm{S}_{\mathrm{f}}= \begin{cases}0 & \text { if } \mathrm{s}_{\mathrm{r}}<0.25 \\ 0.5 & \text { if } \mathrm{s}_{\mathrm{r}}>0.25 \text { and } \mathrm{s}_{\mathrm{r}}<0.75 \\ 1 & \text { if } \mathrm{s}_{\mathrm{r}}>0.75\end{cases}
$$

\subsection{Resource Performance}

The trust is calculated on the performance of the resource. In this resource performance we have taken the network bandwidth and the memory available in the grid resource. The bandwidth is calculated by ration of the bandwidth utilized during job submission to the band-width assigned during the time of registration as shown in Equation 4:

$$
\mathrm{BW}_{\mathrm{f}}=\frac{\mathrm{BW}_{\mathrm{j}}}{\mathrm{BW}_{\mathrm{r}}}
$$

Where:

$\mathrm{BW}_{\mathrm{j}}=$ Total Bandwidth used during job submission

$\mathrm{BW}_{\mathrm{r}}=$ Total Bandwidth assigned by the resource provider during registration

Another performance metric taken from the resource is memory. If the availability of the memory is engaged throughout then the resource is very busy.

In this case we can trust more but the job has to wait for a longer time to use that particular resource. The Equation 5 shows the ratio of the used memory to the total available memory the re-source performance is take for our consideration:

$$
\operatorname{Mem}_{\mathrm{f}}=\frac{\operatorname{Mem}_{\mathrm{u}}}{\mathrm{Mem}_{\mathrm{r}}}
$$

Where:

$\mathrm{Mem}_{\mathrm{u}}=$ The total memory utilized during job submission

$\mathrm{Mem}_{\mathrm{r}}=$ The total memory assigned during registration

All the performance is updated in the trust database for submitting each and every job.

\subsection{Trust Decision}

When the user submits a job through agent (broker), it collects the matching resources available in the grid site. All these matching resources are verified through trust file then the job is dispatched to the resources. The trust $T(i, j)$ is shown in Equation 6.
Let the trust:

$$
T(i, j)=\alpha \operatorname{ST}(i, j)+\beta O T(i, j)
$$

where, $\mathrm{T}(\mathrm{I}, \mathrm{j}) \varepsilon[0,1]$ denotes the trust, $\mathrm{ST}(\mathrm{i}, \mathrm{j}) \varepsilon\{0,1]$ denotes the Subjective trust and OT(i,j) $\varepsilon[0,1]$ Objective trust of entity $i, j$ represented between 0 and 1 . Where 0 represents No Trust and 1 represents Trust. $\alpha$ and $\beta$ are the weights to have the control over the Subjective trust ST $(i, j)$ and Objective Trust OT(i, j). To have a control on weight ST $(i, j)$ and $O T(i, j)$ a function $\phi(j) \varepsilon[0,1]$.

ST $(i, j)$ and Objective Trust OT $(i, j)$. To have a control on weight ST $(i, j)$ and OT $(i, j)$ a function $\phi(j) \varepsilon[0,1]$.

Using all the recommended entries the function $\phi(j)$ is calculated Equation 7:

$$
\phi(j)=1-\frac{1}{R_{e}+\delta}
$$

In expression $7, \delta$ is a positive constant to have a control over $\phi(\mathrm{j})$. The greater constant value $\delta$ will result $\phi(j)$ to closer to 1 , which will decrease $\alpha$ and increase $\beta$ quickly:

$$
\mathrm{T}(\mathrm{i}, \mathrm{j})=\left[\begin{array}{l}
\frac{1}{1+\phi(\mathrm{j})}\left[\left(1-\phi(\mathrm{j})^{\mathrm{k}}\right) * \mathrm{ST}[\mathrm{i}, \mathrm{j}]\right] \\
\left.+\frac{1}{1+\phi(\mathrm{j})}\left[\phi(\mathrm{j})+\phi(\mathrm{j})^{\mathrm{k}}\right) * \mathrm{OT}[\mathrm{i}, \mathrm{j}]\right]
\end{array}\right]
$$

$\mathrm{R}_{\mathrm{e}}$ is the number of recommendation entry from users feedback and resource performance. The number of recommendation entry can be provided when $R_{e} \geq 1$. The recommendation $\mathrm{R}_{\mathrm{e}}$ is taken from Users feedback and Resource Performance, if the trust value satisfies the range of entries in Table 1 then recommend is set to 1 for each entries otherwise 0 .

Equation 9-11 shows the trust value for various conditions $1 . \mathrm{Re}_{\mathrm{e}}=\mathrm{k}, 2 . \mathrm{Re}_{\mathrm{e}}<\mathrm{k}$ and $3 . \mathrm{Re}_{\mathrm{e}}>\mathrm{k}$

$\mathrm{k}$ is a threshold to determine the trust factor. If $\mathrm{Re}_{\mathrm{e}}=$ $\mathrm{k}$, then the recommendation entities became critical and $\mathrm{k}=0$ in Equation 8:

$$
T(i, j)=\frac{1}{1+j(j)}[S T[i, j]+0 T[i, j]]
$$

where, $\phi(\mathrm{j}) \cong 1$

If $\mathrm{Re}_{\mathrm{e}}<\mathrm{k}$, then the threshold entities became critical as $\mathrm{k}=0, \phi(\mathrm{j})$ depends on the recommendation entry and Equation 8:

$$
T(i, j)=\frac{1}{1+j(j)}[S T[i, j]+[j(j) * O T[i, j]]]
$$


Table 1. Recommendation entry

\begin{tabular}{lc}
\hline Recommendation entry $(\mathrm{Re})$ & Range \\
\hline $\mathrm{Aff}_{\mathrm{f}}$ & $>0.25$ \\
$\mathrm{Av}_{\mathrm{f}}$ & $0.25-0.75$ \\
$\mathrm{~S}_{\mathrm{f}}$ & $0.25-0.75$ \\
$\mathrm{BW}_{\mathrm{f}}$ & $<0.75$ \\
$\mathrm{Mem}_{\mathrm{f}}$ & $<0.75$ \\
\hline
\end{tabular}

If $\mathrm{Re}_{\mathrm{e}}>\mathrm{k}$, then the subjective trust will have higher rec-ommendation trust then the Equation 8 becomes:

$$
\begin{aligned}
& \mathrm{T}(\mathrm{i}, \mathrm{j})=\frac{1}{1+\phi(j)}\left[\left[\left(1-\phi(\mathrm{j})^{\mathrm{k}}\right) * \mathrm{ST}[\mathrm{i}, \mathrm{j}]\right]+\right. \\
& \left.\left[\left(\phi(\mathrm{j})+\phi(\mathrm{j})^{\mathrm{k}}\right) * \mathrm{OT}[\mathrm{i}, \mathrm{j}]\right]\right]
\end{aligned}
$$

If there is no interaction between $\mathrm{i}$ and $\mathrm{j}$ then $\alpha=0$ and $\beta=1$ i.e., there is no Subjective Trust ST and we have only Objective Trust OT:

$$
\mathrm{T}(\mathrm{i}, \mathrm{j})=\mathrm{OT}[\mathrm{i}, \mathrm{j}]
$$

If there is more interaction between $\mathrm{i}$ and $\mathrm{j}$ then $\alpha=$ 1 and $\beta=0$ i.e., there is no Objective Trust OT and we have only Subjective Trust ST:

$$
T(i, j)=S T[i, j]
$$

The proposed Trust Resource Algorithm to select the resource in the grid is shown below:

Trust Resource Allocation Algorithm

RヶResource Available

$\mathrm{Job}_{i} \rightarrow$ Job for execution

$\mathrm{Re}_{\mathrm{e}} \leftarrow$ Recommendation Entries

begin

for each user $\mathrm{i}$

job[queue] $=$ jobi $_{i}$

end for

for each jobi $\varepsilon$ job

get the resource $\mathrm{Ri}_{\mathrm{i}}$

if trust_resource ()$\geq 0.5$ then match found submit jobi to $\mathrm{R}_{\mathrm{i}}$ exit()

else if $\mathrm{R}$ exists end if

get the next $\mathrm{R}$ till all $\mathrm{R}$ completes

end for

end if

end

trust_resource $($ )

begin
//Get feedback and resource performance from trust file to calculate trust.

$$
\begin{aligned}
& \text { if } A f f f>0.25 \text { then } A f f f=1 \text { else } A f_{f}=0 \\
& \text { if } A v f>0.25 \text { and } A v f<0.75 \text { then } A v f=1 \text { else } A v f=0 \\
& \text { if } \mathrm{S}_{\mathrm{f}}>0.25 \text { and } \mathrm{S}_{\mathrm{f}}<0.75 \text { then } \mathrm{S}_{\mathrm{f}}=1 \text { else } \mathrm{S}_{\mathrm{f}}=0 \\
& \text { if } \mathrm{BW}_{\mathrm{f}}<0.75 \text { then } \mathrm{BW}_{\mathrm{f}}=1 \text { else } \mathrm{BW}_{\mathrm{f}}=0 \\
& \text { if } \mathrm{Memf}_{\mathrm{f}}<0.75 \text { then } \mathrm{Memf}_{\mathrm{f}}=1 \text { else } \mathrm{Memf}_{\mathrm{f}}=0 \\
& \mathrm{Re}_{\mathrm{e}} \leftarrow \operatorname{Sum}(\mathrm{Aff} f, \mathrm{Avf}, \mathrm{Sf}, \mathrm{BW} \text {, Memf) }
\end{aligned}
$$

$$
\begin{aligned}
& j(j) \leftarrow 1-\frac{1}{R_{e}+\delta} \\
& T(i, j)=\left[\begin{array}{l}
\frac{1}{1+\phi(j)}\left[\left(1-\phi(j)^{k}\right) * S T[i, j]\right. \\
+\frac{1}{1+\phi(j)}\left[\left(\phi(j)+\phi(j)^{k}\right) * O T[i, j]\right]
\end{array}\right] \\
& T(i, j) \leftarrow \alpha S T(i, j)+\beta O T(i, j) \\
& \text { return } T(i, j) ;
\end{aligned}
$$

end

In the above algorithm based on the $\mathrm{Re}_{\mathrm{e}}$ the function $\phi(\mathrm{j})$ is calculated to find the trust $\mathrm{T}(\mathrm{i}, \mathrm{j})$

\section{RESULTS}

In order to evaluate the effect of feedback and resource performance some experiments are designed. In our simulation setup we have considered Intel (R) Core (TM) i3 CPU $2.4 \mathrm{GHz}, 3$ GB Memory, Windows 7 and Gridsim toolkit 4.0. The outcome is compared with and without trust. In our method most of the resources with trusted value is more when compared to without trust. If the load in the grid environment is increased the overall trust is more if we compared to without trust.

\section{DISCUSSION}

In this method for evaluation the trust for the function $\phi$ (j) and Recommendation entry $R_{e}$, we have taken a sample of 500 jobs. For each request of our job we have observed the weight of the trust. As shown in Fig. 2 the weight of most jobs are above 0.5 of the threshold vale $\mathrm{k}$. To increase the weight of trust the value of a constant is raised.

Figure 3 shows that the number of resource available in different general trust. The trust $T(i, j)$ is calculated for all available jobs. A comparative is done for two different categories 1 . Without trust and 2 With trust. For the 500 jobs of input the approximation of 6 sec is taken. In this duration we have observed that only an average of 50 resources are available for each time, where as if we have the trusted resource then the number of resource available is varied. 
Prakash Mohan and Ravichandran Thangavel / American Journal of Applied Sciences 10 (8): 924-930, 2013

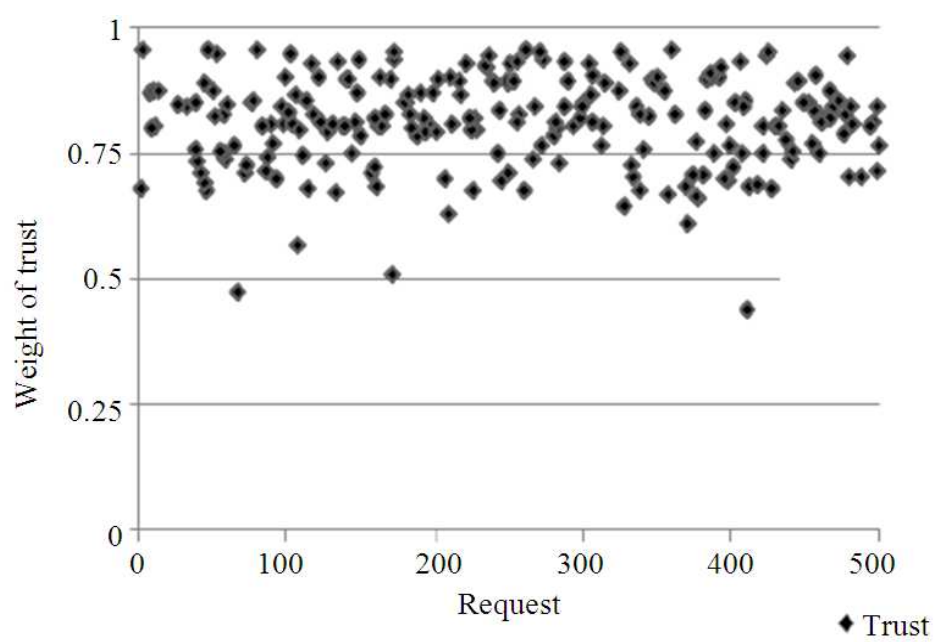

Fig. 2. Weight of trust

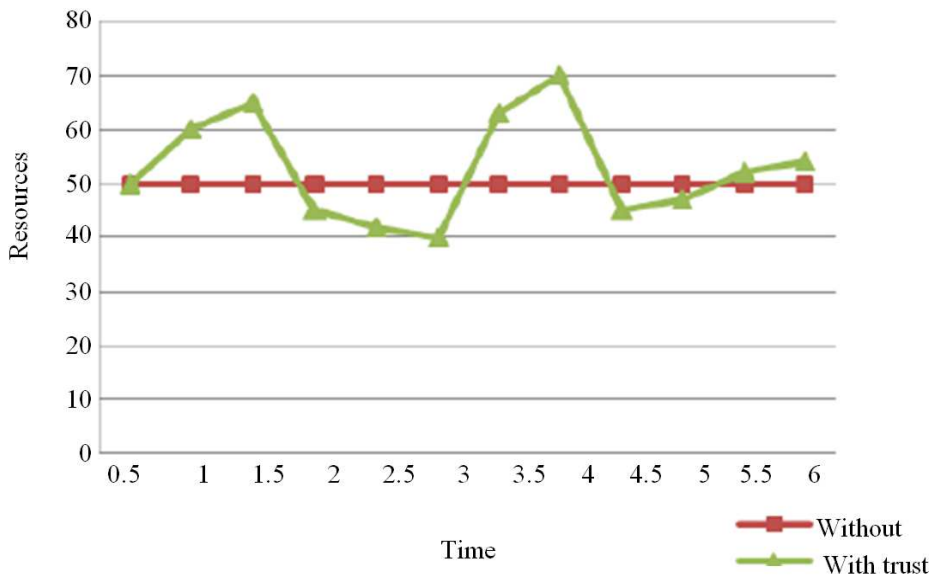

Fig. 3. Number of resource available in different trust

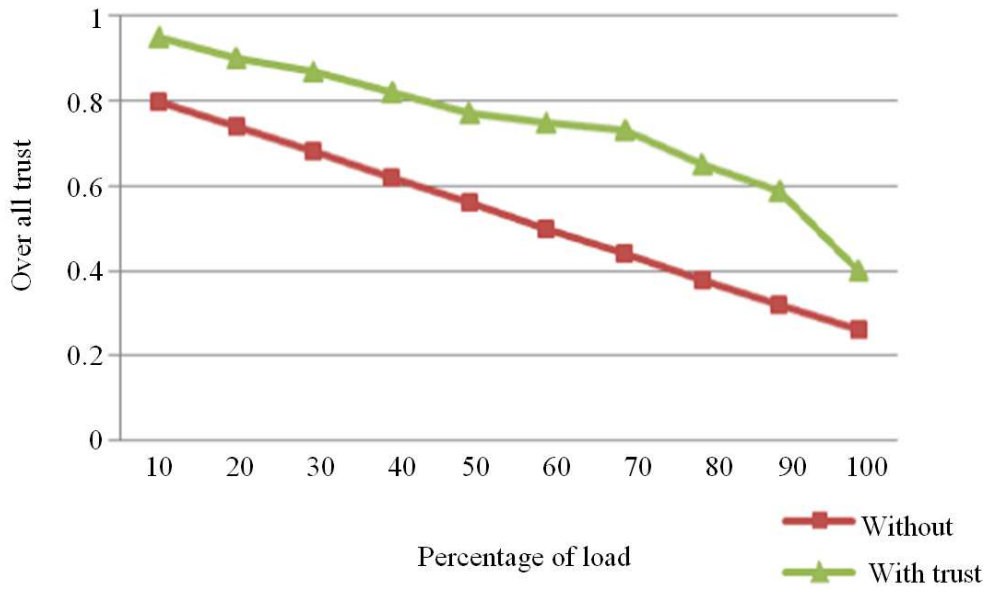

Fig. 4. Overall trust value of resource 
In the grid environment if the load increases then the job schedule is unbalanced, at this point the task is scheduled to the available resource in grid. We have tested if the load increased gradually then the overall trust value in the resource is decreased. In Fig. 4 it show clearly the increase in load leads to a gradual decrease in overall trust.

\section{CONCLUSION}

In this study, we presented how to select the trust resource. As soon as the job is completed by the resource the trusted file is updated as feedback and the resource performance given by the user. When the user submits the job, the agent matches the jobs with the help of trust file to find the trustworthy of the resource. The result from the simulation shows that the trustworthiness of our model based on the weight of trust. In the other way in our system we can submit more number of jobs in our model because for the trusted weight we can utilize more number of jobs.

\section{REFERENCES}

Aron, R. and I. Chan, 2012. Formal QoS policy based grid resource provisioning framework. J. Grid Comput., 10: 249-264. DOI: 10.1007/s10723-0129202-y

Bawa, R.K. and G. Sharma, 2012. Reliable resource selection in grid environment. Int J. Grid Comput. Applic., 3: 1-10.

Can, A.B. and B. Bhargava, 2013. SORT: A selforganizing trust model for peer-to-peer systems. IEEE Trans. Dependable Secure Comput., 10: 1427. DOI: 10.1109/TDSC.2012.74
Foster, I., C. Kesselman and S. Tuecke, 2001. The anatomy of the grid: Enabling scalable virtual organizations. Int. J. High Performance Comput. Applic., 15: 200-222. DOI: 10.1177/109434200101500302

Li, M. and M. Baker, 2005. The Grid: Core Technologies. 1st Edn., John Wiley and Sons, Chichester, ISBN-10: 0470094176, pp: 452.

McKnight, D.H. and N.L. Chervany, 2001. Trust and Distrust Definition: One Bite at a Time. In: Trust in Cyber-Societies, Falcone, R., M. Singh and Y.H. Tan (Eds.), Springer, Berlin, ISBN-10: 3540430695 , pp: 27-54.

Shun-Fu, X., W., Bing, Z. Xue-Jie, Z. Shi-Ming, S. Rong-Ming, 2012. Research on trustworthinessbased grid resource selection optimized algorithm. Proceedings of the International Conference on Informatics, Cybernetics and Computer Engineering, Nov. 19-20, Melbourne, Australia, pp: 627-635. DOI: 10.1007/978-3-642-25185-6_80

Siadat, S., A.M. Rahmani and M. Mohsenzadeh, 2009. Proposed platform for improving grid security by trust management system. Int. J. Comput. Sci. Inform. Sec., 6: 143-148.

Wang, L.J., G.H. Zhang and S. Chen, 2011. A Trust Model Under Grid Computing Environment. In: Information Engineering and Applications, Zhu, R. and Y. Ma (Eds.), Springer, London, ISBN-10: 1447123867, pp: 420-427. 